\title{
A note on the transmission feasibility problem in networks
}

https://doi.org/10.1515/phys-2018-0110

Received November 16, 2018; accepted November 23, 2018

\begin{abstract}
In the networking designing phase, the network needs to be built according to certain indicators to ensure that the network has the ideal functions and can work smoothly. From a modeling perspective, each site in the network is represented by a vertex, channels between sites are represented by edges, and thus the entire network can be denoted as a graph. Problems in the network can be transformed into corresponding graph problems. In particular, the feasibility of data transmission can be transformed into the existence of fractional factors in network graph. This note gives an independent set neighborhood union condition for the existence of fractional factors in a special setting, and shows that the neighborhood union condition is sharp.
\end{abstract}

Keywords: Data transmission network, fractional factor, independent set, neighborhood union

PACS: 02.10.0x, 07.05.Mh

\section{Introduction}

In the initial stage of network designing, scientists pre-conceive what requirements the built network needs to meet, and then plan the structure of the network as needed, including the distribution of the sites and the channels between the sites. In mathematical modelling, each site is represented by a vertex, and the channel connecting the two sites is represented by an edge. The direction of the edge is defined according to the direction of information flow between the two sites, and the undirected edge implies two-directional flow between the two sites.

\footnotetext{
Wei Gao: Department of Mathematics, Nanjing University, Nanjing 210093, China, E-mail: gaowei@ynnu.edu.cn Yunqing Zhang: Department of Mathematics, Nanjing University, Nanjing 210093, China, E-mail: yunqingzh@nju.edu.cn

*Corresponding Author: Yaojun Chen: Department of Mathematics, Nanjing University, Nanjing 210093, China,

E-mail: yaojunc@nju.edu.cn
}

As a result, problems in the network, in particular problems in the network designing phase, can be described by the problems in a network graph. In the design of the transmission network, we always require the network as a whole to achieve the pre-conceived transmission requirements, especially when some channels are not working properly due to failure or being under attacked for some unexpected reasons, the entire network is unobstructed. The problem of the transmission of data packets within a certain range within the network is equivalent to the existence of the fractional factor, from the graph theory point of view. In addition, the smooth procedure transmission under the condition that some channels that can't are not work is equivalent to the fractional deleted graph problem in graph theory. That is, after deleting given number of edges, the fractional factor still exists.

In this note, we discuss the theoretical problem from graph theory point of view, and consider each network as a simple graph (loopless, finite multiple edges). Let $G$ be a graph with vertex set $V(G)$ and edge set $E(G)$. Some fundamental notations are listed as follows (set $S \subseteq V(G)$ ):

- $d_{G}(x)$ : degree of vertex $x$ in $G$. For simply, we write it as $d(x)$;

- $N_{G}(x)$ (in short, $N(x)$ ): neighborhood of $x$ in $G$;

- $N_{G}[x]: N_{G}(x) \cup\{x\}$;

- $G[S]$ : subgraph of $G$ which is induced by $S$;

- $G-S: G[V(G) \backslash S]$;

- $\delta(G)$ : minimum degree $G$;

- $n$ : order of graph, i.e., $n=|V(G)|$;

- a matching $M$ of $G$ : a subset of $E(G)$ such that no two elements are adjacent;

- $e_{G}(S, T)$ (here $S$ and $T$ are vertex-disjoint subsets of $G$ ): $|\{e=x y \mid x \in S, y \in T\}|$.

The terminologies used in this paper but undefined here can be referred to Bondy and Murty [1].

Assume that $g$ and $f$ are two integer-valued functions on $V(G)$ satisfy $0 \leq g(x) \leq f(x)$ for any $x \in V(G)$. A fractional $(g, f)$-factor is a function $h$ that assigns a number in $[0,1]$ to each edge of a graph $G$ a number in $[0,1]$, so that $g(x) \leq \sum_{e \in E(x)} h(e) \leq f(x)$ for each vertex $x$. A fractional $(g, f)$-factor is called a fractional $[a, b]$-factor if $g(x)=a$ and $f(x)=b$ for any $x \in V(G)$. Moreover, a fractional $(g, f)$ - 
factor is called a fractional $f$-factor if $g(x)=f(x)$ for all $x \in V(G)$.

Anstee [2] determined the necessary and sufficient condition for the existence of a fractional $(g, f)$-factor which is manifested as follows.

Theorem 1. (Anstee [2]) Let $G$ be a graph, and let $g, f$ : $V(G) \rightarrow\{0,1,2, \cdots\}$ be two functions with $g(x) \leq f(x)$ for each $x \in V(G)$. Then $G$ has a fractional $(g, f)$-factor if, and only if,

$$
f(S)+d_{G-S}(T)-g(T) \geq 0
$$

for any $S \subseteq V(G)$ and $T=\left\{x: x \in V(G)-S, d_{G-S}(x) \leq\right.$ $g(x)\}$.

Let $S \subseteq V(G), M$ be a matching, $T=\{x: x \in V(G)$ $\left.S, d_{G-S}(x) \leq g(x)\right\}, D=V(G) \backslash(S \cup T), E_{G}(T)=\{e: e=x y \in$ $E(G), x, y \in T\}, E^{\prime}=M \cap E_{G}(T), E^{\prime \prime}=M \cap E_{G}(T, D)$ and $\beta_{G}(S, T, M)=2\left|E^{\prime}\right|+\left|E^{\prime \prime}\right|$. Li et al. [3] derived a necessary and sufficient condition for a graph to have a fractional $(g, f)$-factor excluding a given matching $M$ (i.e., $h(e)=0$ for any edge in $M$ ).

Theorem 2. (Li et al. [3]) Let $G$ be a graph, $g, f: V(G) \rightarrow$ $\{0,1,2, \cdots\}$ be two functions with $g(x) \leq f(x)$ for each $x \in V(G)$, and $M$ be a matching of $G$. Then $G$ has a fractional $(g, f)$-factor excluding $M$ if, and only if,

$$
f(S)+d_{G-S}(T)-g(T) \geq \beta_{G}(S, T, M)
$$

for any $S \subseteq V(G)$ and $T=\left\{x: x \in V(G)-S, d_{G-S}(x) \leq\right.$ $g(x)\}$.

In recent years, the problem of fractional factor has raised attention in both graph theory and computer networks. Gao et al. [4], [5], [6] and [7] studied the relationship between toughness (or isolated toughness) and the existence of fractional factor in different settings. Gao et al. [8] and [9] determined several independent set conditions for a graph with fractional factors. Furthermore, neighborhood conditions for the existence of fractional factors in special settings were researched in Gao et al. [10], [11] and [12]. More results on fractional factors can be referred to Zhou et al. [13], [14], [15], [16], [17], and [18], Khellat and Khormizi [19], Lokesha et al. [20], and Pandey and Jaboob [21].

A matching is called $m$-matching if it contains $m$ edges. The aim of this note is to study the relationship between independent set neighborhood union characteristics and the existence of fractional factors in a special setting where a $m$-matching is deleted from $G$. In computer networks, neighborhood union reflects the degree of density in networks, and independent set neighborhood union reflects the distribution of the dominant set in networks. The main contribution in our work is stated in the following theorem.
Theorem 3. Let $G$ be a noncomplete graph of order $n$. Let $a, b, m, \Delta$ and $i$ be nonnegative integers with $i \geq 2$, $2 \leq a \leq b-\Delta$. Let $g, f$ be two integer-valued functions defined on $V(G)$ such that $a \leq g(x) \leq f(x)-\Delta \leq b-\Delta$ for each $x \in V(G)$. Let $e$ be an edge of $G$, and $M$ be an $m$-matching of $G$ satisfying $e / \in M$. If $\delta(G) \geq \frac{(i-1)(b-\Delta)(b+2)+1}{a+\Delta}+1, n \geq$ $\frac{(a+b)(i(a+b+1)+2 m-2)+2}{a+\Delta}$, and

$$
\left|N_{G}\left(x_{1}\right) \cup N_{G}\left(x_{2}\right) \cup \cdots \cup N_{G}\left(x_{i}\right)\right| \geq \frac{(b-\Delta) n+2}{a+b}+i
$$

for any independent subset $\left\{x_{1}, x_{2}, \cdots, x_{i}\right\}$ of $V(G)$, then $G$ has a fractional $(g, f)$-factor containing $e$ and excluding M.

If $m=1$ in Theorem 3, then we get the following corollary.

Corollary 1. Let $G$ be a noncomplete graph of order $n$. Let $a, b, m, \Delta$ and $i$ be nonnegative integers with $i \geq 2$, $2 \leq a \leq b-\Delta$. Let $g, f$ be two integer-valued functions defined on $V(G)$, such that $a \leq g(x) \leq f(x)-\Delta \leq b-\Delta$ for each $x \in V(G)$. Let $e_{1}$ and $e_{2}$ be two distinct edges of $G$. If $\delta(G) \geq \frac{(i-1)(b-\Delta)(b+2)+1}{a+\Delta}+1, n \geq \frac{(a+b)(i(a+b+1))+2}{a+\Delta}$, and

$$
\left|N_{G}\left(x_{1}\right) \cup N_{G}\left(x_{2}\right) \cup \cdots \cup N_{G}\left(x_{i}\right)\right| \geq \frac{(b-\Delta) n+2}{a+b}+i
$$

for any independent subset $\left\{x_{1}, x_{2}, \cdots, x_{i}\right\}$ of $V(G)$, then $G$ has a fractional $(g, f)$-factor containing $e_{1}$ and excluding $e_{2}$.

If we set $\Delta=0$ in Theorem 3, then the following corollary is obtained.

Corollary 2. Let $G$ be a noncomplete graph of order $n$. Let $a, b, m$ and $i$ be nonnegative integers with $i \geq 2,2 \leq a \leq b$. Let $g, f$ be two integer-valued functions defined on $V(G)$, such that $a \leq g(x) \leq f(x) \leq b$ for each $x \in V(G)$. Let $e$ be an edge of $G$ and $M$ be an $m$-matching of $G$ satisfying $e / \in M$. If $\delta(G) \geq \frac{(i-1) b(b+2)+1}{a}+1, n \geq \frac{(a+b)(i(a+b+1)+2 m-2)+2}{a}$, and

$$
\left|N_{G}\left(x_{1}\right) \cup N_{G}\left(x_{2}\right) \cup \cdots \cup N_{G}\left(x_{i}\right)\right| \geq \frac{b n+2}{a+b}+i
$$

for any independent subset $\left\{x_{1}, x_{2}, \cdots, x_{i}\right\}$ of $V(G)$, then $G$ has a fractional $(g, f)$-factor containing $e$ and excluding $M$.

If $g(x)=f(x)$ for any $x \in V(G)$ in Corollary 2, then we yield the following conclusion.

Corollary 3. Let $G$ be a noncomplete graph of order $n$. Let $a, b, m$ and $i$ be nonnegative integers with $i \geq 2,2 \leq a \leq b$. Let $f$ be an integer-valued function defined on $V(G)$, such that $a \leq f(x) \leq b$ for each $x \in V(G)$. Let $e$ be an edge of $G$ and $M$ be an $m$-matching of $G$ satisfying $e / \in M$. If $\delta(G) \geq$ $\frac{(i-1) b(b+2)+1}{a}+1, n \geq \frac{(a+b)(i(a+b+1)+2 m-2)+2}{a}$, and

$$
\left|N_{G}\left(x_{1}\right) \cup N_{G}\left(x_{2}\right) \cup \cdots \cup N_{G}\left(x_{i}\right)\right| \geq \frac{b n+2}{a+b}+i
$$


for any independent subset $\left\{x_{1}, x_{2}, \cdots, x_{i}\right\}$ of $V(G)$, then $G$ has a fractional $f$-factor containing $e$ and excluding $M$.

Let

$$
\varepsilon(S, T)= \begin{cases}2, & S \text { is not an independent set } \\ 1, & S \text { is an independent set and } \\ & e_{G}(S, V(G) \backslash(S \cup T)) \geq 1, \text { or there is } \\ & \text { an edge } e=u v \text { joining } S \text { and } T \text { such } \\ & \text { that } d_{G-S}(v)=g(v) \text { for } v \in T \\ 0, & \text { otherwise. }\end{cases}
$$

To prove Theorem 3, we need the following result.

Theorem 4. (Li et al. [22]) Let $G$ be a graph, and let $g, f$ : $V(G) \rightarrow \mathbb{N} \cup\{0\}$ be two functions such that $g(x) \leq f(x)$ for every $x \in V(G)$. Then $G$ has a fractional $(g, f)$-factor containing any given edge $e$ if, and only if,

$$
f(S)+d_{G-S}(T)-g(T) \geq \varepsilon(S, T)
$$

for any vertex subset $S$ of $G$ and $T=\{x: x \in V(G)$ $\left.S, d_{G-S}(x) \leq g(x)\right\}$.

\section{Proof of Theorem 3}

Let $H=G-M$, and we have $V(H)=V(G)$ and $\delta(H) \geq$ $\delta(G)-1$. It is enough to show that $H$ has a fractional $(g, f)$ factor including $e$. In what follows, we use the counterevidence method to prove this result. Assume that $H$ does't have the desired fractional factor. Then in terms of Theorem 4, there exists a subset $S \subseteq V(H)$ that satisfies

$$
f(S)+d_{H-S}(T)-g(T) \leq \varepsilon(S, T)-1
$$

where $T=\left\{x: x \in V(H)-S, d_{H-S}(x) \leq g(x)\right\}$. If $T=\emptyset$, then $d_{H-S}(T)-g(T)=0$ and by (1) we infer $\varepsilon(S, T)-1 \geq f(S) \geq$ $(a+\Delta)|S| \geq 2|S| \geq \varepsilon(S, T)$, a contradiction. Thus, we have $T \neq \emptyset$.

Set $D=V(G)-S \cup T$ and $E_{G}(T)=\{e: e=x y \in$ $E(G), x, y \in T\}$. Since $M$ is an $m$-matching, we deduce

$$
\begin{aligned}
& d_{H-S}(T)=d_{G-M-S}(T) \\
= & d_{G-S}(T)-\left(2\left|M \cap E_{G}(T)\right|+\left|M \cap E_{G}(T, D)\right|\right) \\
\geq & d_{G-S}(T)-\min \{2 m,|T|\} .
\end{aligned}
$$

Now, we discuss the following three cases according to the value of $|S|$.

Case 1. $|S|=0$.

In this case, we have $\varepsilon(S, T)=0$ and $\delta(H) \geq \delta(G)-1 \geq$ $\frac{(i-1)(b-\Delta)(b+2)+1}{a}>b-\Delta$. By means of (1), we yield

$$
\begin{aligned}
& -1 \geq f(S)+d_{H-S}(T)-g(T) \\
=\quad & d_{H}(T)-g(T)=\sum_{x \in T}\left(d_{H}(x)-g(x)\right)
\end{aligned}
$$

$$
\geq \sum_{x \in T}(\delta(H)-(b-\Delta)) \geq 0,
$$

a contradiction.

Case 2. $|S|=1$.

In this case, we have $\varepsilon(S, T) \leq 1$ and $\delta(G) \geq$ $\frac{(i-1)(b-\Delta)(b+2)+1}{a}+1>b-\Delta+1$. In view of the integrity of $\delta(G)$, we have $\delta(G) \geq b-\Delta+2$. In light of (1) and (2), we derive

$$
\begin{aligned}
& 0 \geq \varepsilon(S, T)-1 \geq f(S)+d_{H-S}(T)-g(T) \\
\geq & f(S)+d_{G-S}(T)-\min \{|T|, 2 m\}-g(T) \\
\geq & f(S)+d_{G-S}(T)-|T|-g(T) \\
\geq & f(S)+d_{G}(T)-2|T|-g(T) \\
\geq & f(S)+\delta(G)|T|-2|T|-g(T) \\
\geq & (a+\Delta)|S|+(b-\Delta+2)|T|-2|T|-(b-\Delta)|T| \\
= & (a+\Delta)|S|=a+\Delta \geq 2,
\end{aligned}
$$

a contradiction.

\section{Case 3. $|S| \geq 2$.}

Note that $\varepsilon(S, T) \leq 2$ and $T \neq \emptyset$. Let $d_{1}=\min \left\{d_{G-S}(x)\right.$ : $x \in T\}$ and choose $x_{1} \in T$ such that $d_{G-S}\left(x_{1}\right)=d_{1}$. If $T-N_{T}\left[x_{1}\right] \neq \emptyset$, let $d_{2}=\min \left\{d_{G-S}(x): x \in T-N_{T}\left[x_{1}\right]\right\}$ and choose $x_{2} \in T-N_{T}\left[x_{1}\right]$ such that $d_{G-S}\left(x_{2}\right)=d_{2}$. Continue this step, if $z \geq 2$ and $T \backslash\left(\cup_{j=1}^{z-1} N_{T}\left[x_{j}\right]\right) \neq \emptyset$, let

$$
d_{z}=\min \left\{d_{G-S}(x) \mid x \in T \backslash\left(\cup_{j=1}^{z-1} N_{T}\left[x_{j}\right]\right)\right\},
$$

and select $x_{z} \in T \backslash\left(\cup_{j=1}^{z-1} N_{T}\left[x_{j}\right]\right)$ with $d_{G-S}\left(x_{z}\right)=d_{z}$. Thus, we construct a sequence $0 \leq d_{1} \leq d_{2} \leq \cdots \leq d_{\pi} \leq b-\Delta+1$ and an independent set $\left\{x_{1}, x_{2}, \cdots, x_{\pi}\right\} \subseteq T$.

Now, we the next lemma which is similar to Lemma 5 in Gao et al. [8].

Lemma 1. In the above conditions, we have

$$
|T| \geq \begin{cases}(i-1)(b+2), & \text { if } d_{G-S}(x)=1 \text { for any } x \in T, \\ (i-1)(b+2)+1, & \text { otherwise. }\end{cases}
$$

Proof. Note that $|S|+d_{1}=|S|+d_{G-S}\left(x_{1}\right) \geq d_{G}\left(x_{1}\right) \geq \delta(G)$. We verify that

$$
\begin{array}{ll} 
& |S| \geq \delta(G)-d_{1} \\
\geq & \frac{(i-1)(b-\Delta)(b+2)+1}{a+\Delta}+1-d_{1} .
\end{array}
$$

Firstly, we justify that $|T| \geq(i-1)(b+2)$ if $d_{G-S}(x)=1$ for any $x \in T$. In this case, we get $d_{1}=1$.

Assume that $|T| \leq(i-1)(b+2)-1$. By means of (2), (3), $d_{1}=1$ and $i \geq 2$, we yield

$$
\begin{aligned}
& f(S)+d_{H-S}(T)-g(T) \\
\geq & f(S)+d_{G-S}(T)-\min \{|T|, 2 m\}-g(T) \\
\geq & f(S)+d_{G-S}(T)-|T|-g(T)
\end{aligned}
$$




$$
\begin{aligned}
\geq & (a+\Delta)|S|+|T|-|T|-(b-\Delta)|T| \\
= & (a+\Delta)|S|-(b-\Delta)|T| \\
\geq & (a+\Delta)\left(\frac{(i-1)(b-\Delta)(b+2)+1}{a+\Delta}+1-d_{1}\right) \\
& -(b-\Delta)((i-1)(b+2)-1) \\
= & (a+\Delta) \frac{(i-1)(b-\Delta)(b+2)+1}{a+\Delta} \\
& -(b-\Delta)((i-1)(b+2)-1) \\
= & b-\Delta+1>a \geq 2 \geq \varepsilon(S, T),
\end{aligned}
$$

which contradicts (1). Therefore, the first part of Lemma 1 is established.

Next, we check that $|T| \geq(i-1)(b+2)+1$ in the rest cases. Assume that $|T| \leq(i-1)(b+2)$ in this case, and the discussion can be divided into three situations in terms of the value of $d_{1}$.

Case 1. $d_{1}=0$.

In view of (1), (3) and $d_{1}=0$, we yield

$$
\begin{aligned}
& \varepsilon(S, T)-1 \geq f(S)+d_{H-S}(T)-g(T) \\
\geq & f(S)-g(T) \geq(a+\Delta)|S|-(b-\Delta)|T| \\
\geq & (a+\Delta)\left(\frac{(i-1)(b-\Delta)(b+2)+1}{a+\Delta}+1-d_{1}\right) \\
& -(b-\Delta)(i-1)(b+2) \\
= & a+\Delta+1>a \geq 2 \geq \varepsilon(S, T),
\end{aligned}
$$

a contradiction.

Case 2. $d_{1}=1$.

Following from (1), (3), $d_{1}=1$ and $\varepsilon(S, T) \leq 2$, we get

$$
\begin{array}{ll} 
& 1 \geq \varepsilon(S, T)-1 \geq f(S)+d_{H-S}(T)-g(T) \\
\geq \quad & f(S)-g(T) \geq(a+\Delta)|S|-(b-\Delta)|T| \\
\geq \quad & (a+\Delta)\left(\frac{(i-1)(b-\Delta)(b+2)+1}{a+\Delta}+1-d_{1}\right) \\
& -(b-\Delta)(i-1)(b+2)
\end{array}
$$$$
=1 \text {. }
$$

It implies that all the " $\geq$ " should be "=", and it further reveals that $d_{H-S}(T)=0$, i.e., $d_{H-S}(x)=0$ for each $x \in T$.

Since $d_{1}=1$, we confirm that $d_{G-S}(x)=1$ for each $x \in T$ which contradicts the assumption that there exist $x \in T$ satisfying $d_{G-S}(x) \neq 1$.

Case 3. $2 \leq d_{1} \leq b-\Delta+1$.

Using (1), (2), (3), $i \geq 2$ and $2 \leq d_{1} \leq b-\Delta+1$, we derive

$$
\begin{aligned}
& \varepsilon(S, T)-1 \geq f(S)+d_{H-S}(T)-g(T) \\
\geq & f(S)+d_{G-S}(T)-\min \{|T|, 2 m\}-g(T) \\
\geq & f(S)+d_{G-S}(T)-|T|-g(T) \\
\geq & (a+\Delta)|S|+d_{1}|T|-|T|-(b-\Delta)|T| \\
= & (a+\Delta)|S|-\left(b-\Delta-d_{1}+1\right)|T| \\
\geq & (a+\Delta)\left(\frac{(i-1)(b-\Delta)(b+2)+1}{a+\Delta}+1-d_{1}\right)
\end{aligned}
$$

$$
\begin{aligned}
& -\left(b-\Delta-d_{1}+1\right)(i-1)(b+2) \\
= & \left(d_{1}-1\right)((i-1)(b+2)-(a+\Delta))+1 \\
\geq \quad & \left(d_{1}-1\right)((b+2)-(a+\Delta))+1 \\
>\quad & \left(d_{1}-1\right)+1=d_{1} \\
\geq & 2 \geq \varepsilon(S, T),
\end{aligned}
$$

a contradiction.

Therefore, we infer that $|T| \geq(i-1)(b+2)+1$ in other cases.

The next lemma shows that the desired independent subset exists in $T$.

Lemma 2. In the previous setting, there exists an independent subset $\left\{x_{1}, x_{2}, \cdots, x_{i}\right\} \subseteq T$.

Proof. If $d_{G-S}(x)=1$ for any $x \in T$, then in terms of Lemma 1, we have $|T| \geq(i-1)(b+2)$. In light of $b \geq 2$ and $d_{G-S}(x)=1$ for any $x \in T$, we determine that $d_{G-S}(x) \leq b-1$ for any $x \in T$. Combining this with $|T| \geq(i-1)(b+2)=$ $b(i-1)+2(i-1) \geq b(i-1)+2$, we conclude that there exists an independent subset $\left\{x_{1}, x_{2}, \cdots, x_{\pi}\right\} \subseteq T$ for $\pi=i$.

In other cases, it holds that $|T| \geq(i-1)(b+2)+1$ by means of Lemma 1. It is clear that $d_{G-S}(x) \leq d_{H-S}(x)+1 \leq$ $g(x)+1 \leq b-\Delta+1 \leq b+1$ for any $x \in T$. Thus, we can find an independent subset, $\left\{x_{1}, x_{2}, \cdots, x_{\pi}\right\} \subseteq T$ for $\pi=i$.

The proof of Lemma 2 is completed.

By lemma 2 and the condition of Theorem 3, we have

$$
\begin{aligned}
& \frac{(b-\Delta) n+2}{a+b}+i \\
\leq & \left|N_{G}\left(x_{1}\right) \cup N_{G}\left(x_{2}\right) \cup \cdots \cup N_{G}\left(x_{i}\right)\right| \\
\leq & |S|+\sum_{j=1}^{i} d_{j}
\end{aligned}
$$

and

$$
|S| \geq \frac{(b-\Delta) n+2}{a+b}+i-\sum_{j=1}^{i} d_{j} .
$$

The next lemma presents the lower bound of $d_{i}$.

Lemma 3. $d_{i} \geq 2$.

Proof. Suppose that $d_{i}=0$ or $d_{i}=1$. In terms of (4) and $|S|+|T| \leq n$, we ensure that

$$
\begin{aligned}
& f(S)+d_{H-S}(T)-g(T) \\
\geq & f(S)-g(T) \\
\geq & (a+\Delta)|S|-(b-\Delta)|T| \\
\geq & (a+\Delta)|S|-(b-\Delta)(n-|S|) \\
= & (a+b)|S|-(b-\Delta) n \\
\geq & (a+b)\left(\frac{(b-\Delta) n+2}{a+b}+i-\sum_{j=1}^{i} d_{j}\right) \\
& -(b-\Delta) n \\
\geq & (a+b) \frac{(b-\Delta) n+2}{a+b}-(b-\Delta) n
\end{aligned}
$$


$=2 \geq \varepsilon(S, T)$,

which contradicts (1). Hence we get $d_{i} \in\{0,1\}$.

It is easy to check that

$$
\begin{aligned}
& \left|N_{T}\left[x_{j}\right]\right|-\left|N_{T}\left[x_{j}\right] \cap\left(\cup_{z=1}^{j-1} N_{T}\left[x_{z}\right]\right)\right| \\
\geq \quad & 1, j=2,3, \cdots, i-1, \\
& \left|\cup_{z=1}^{j} N_{T}\left[x_{z}\right]\right| \leq \sum_{z=1}^{j}\left|N_{T}\left[x_{z}\right]\right| \\
\leq & \sum_{z=1}^{j}\left(d_{G-S}\left(x_{z}\right)+1\right) \\
= & \sum_{z=1}^{j}\left(d_{z}+1\right), j=1,2, \cdots, i .
\end{aligned}
$$

and

$$
|T|-\left|\cup_{j=1}^{i} N_{T}\left[x_{j}\right]\right| \geq 1 .
$$

It follows from (1), (2), (5), (6), $n \geq|S|+|T|$, Lemma 3 and $d_{i} \leq b-\Delta+1$ that

$$
\begin{aligned}
& 1 \geq \varepsilon(S, T)-1 \\
& \geq f(S)+d_{H-S}(T)-g(T) \\
& \geq f(S)+d_{G-S}(T)-\min \{|T|, 2 m\}-g(T) \\
& \geq f(S)+d_{G-S}(T)-g(T)-2 m \\
& \geq \quad(a+\Delta)|S|-(b-\Delta)|T|+d_{1}\left|N_{T}\left[x_{1}\right]\right| \\
& +d_{2}\left(\left|N_{T}\left[x_{2}\right]\right|-\left|N_{T}\left[x_{2}\right] \cap N_{T}\left[x_{1}\right]\right|\right)+\cdots \\
& +d_{i-1}\left(\left|N_{T}\left[x_{i-1}\right]\right|-\left|N_{T}\left[x_{i-1}\right] \cap\left(\cup_{j=1}^{i-2} N_{T}\left[x_{j}\right]\right)\right|\right) \\
& \left.+d_{i}\left(|T|-\mid \cup_{j=1}^{i-1} N_{T}\left[x_{j}\right]\right) \mid\right)-2 m \\
& \geq \quad(a+\Delta)|S|-(b-\Delta)|T|+d_{1}\left|N_{T}\left[x_{1}\right]\right| \\
& +d_{2}\left(\left|N_{T}\left[x_{2}\right]\right|-\left|N_{T}\left[x_{2}\right] \cap N_{T}\left[x_{1}\right]\right|\right)+\cdots \\
& +d_{i-1}\left(\left|N_{T}\left[x_{i-1}\right]\right|-\left|N_{T}\left[x_{i-1}\right] \cap\left(\cup_{j=1}^{i-2} N_{T}\left[x_{j}\right]\right)\right|\right) \\
& \left.+\left(d_{i}-1\right)\left(|T|-\mid \cup_{j=1}^{i-1} N_{T}\left[x_{j}\right]\right)||\right)-2 m+1 \\
& \geq(a+\Delta)|S|+\left(d_{1}-d_{i}+1\right)\left|N_{T}\left[x_{1}\right]\right|+\sum_{j=2}^{i-1} d_{j} \\
& -\left(b-\Delta+1-d_{i}\right)|T|-\left(d_{i}-1\right) \sum_{j=2}^{i-1}\left|N_{T}\left[x_{j}\right]\right| \\
& -2 m+1 \\
& \geq \quad(a+\Delta)|S|+\left(d_{1}-d_{i}\right)\left(d_{1}+1\right)+1+\sum_{j=2}^{i-1} d_{j} \\
& -\left(b-\Delta+1-d_{i}\right)|T|-\left(d_{i}-1\right) \sum_{j=2}^{i-1}\left(d_{j}+1\right) \\
& -2 m+1
\end{aligned}
$$

$$
\begin{aligned}
= & (a+\Delta)|S|+d_{1}\left(d_{1}-1\right)+\sum_{j=1}^{i-1} d_{j}-2 m+1 \\
& -\left(d_{i}-1\right) \sum_{j=1}^{i-1}\left(d_{j}+1\right)-\left(b-\Delta+1-d_{i}\right)|T| \\
\geq & (a+\Delta)|S|+d_{1}\left(d_{1}-1\right)+\sum_{j=1}^{i-1} d_{j}-2 m+1 \\
& -\left(d_{i}-1\right) \sum_{j=1}^{i-1}\left(d_{j}+1\right)-\left(b-\Delta+1-d_{i}\right)(n-|S|) \\
= & \left(a+b+1-d_{i}\right)|S|+d_{1}\left(d_{1}-1\right)+\sum_{j=1}^{i-1} d_{j}-2 m \\
& -\left(d_{i}-1\right) \sum_{j=1}^{i-1}\left(d_{j}+1\right)-\left(b-\Delta+1-d_{i}\right) n+1,
\end{aligned}
$$

which implies

$$
\left(a+b+1-d_{i}\right)|S|+d_{1}\left(d_{1}-1\right)+\sum_{j=1}^{i-1} d_{j}
$$

$$
-\left(d_{i}-1\right) \sum_{j=1}^{i-1}\left(d_{j}+1\right)-\left(b-\Delta+1-d_{i}\right) n-2 m \leq 0 .
$$

By means of (4), (8), Lemma 3, $i \geq 2, d_{1} \leq d_{2} \leq \cdots \leq$ $d_{i} \leq b-\Delta+1$ and $n \geq \frac{(a+b)(i(a+b+1)+2 m-2)+2}{a+\Delta}$, we have

$$
\begin{aligned}
0 \quad & \left(a+b+1-d_{i}\right)|S|+d_{1}\left(d_{1}-1\right)+\sum_{j=1}^{i-1} d_{j} \\
& -\left(d_{i}-1\right) \sum_{j=1}^{i-1}\left(d_{j}+1\right)-\left(b-\Delta+1-d_{i}\right) n-2 m \\
\geq & \left(a+b+1-d_{i}\right)\left(\frac{(b-\Delta) n+2}{a+b}+i-\sum_{j=1}^{i} d_{j}\right) \\
& -\left(d_{i}-2\right) \sum_{j=1}^{i-1} d_{j}-\left(d_{i}-1\right)(i-1) \\
& -n\left(b-\Delta+1-d_{i}\right)-2 m \\
\geq & \left(a+b+1-d_{i}\right)\left(\frac{(b-\Delta) n+2}{a+b}+i-i d_{i}\right) \\
& -\left(d_{i}-2\right) d_{i}(i-1)-\left(d_{i}-1\right)(i-1) \\
& -n\left(b-\Delta+1-d_{i}\right)-2 m \\
= & \left(d_{i}-1\right)\left(\frac{(a+\Delta) n-2}{a+b}-i(a+b+1)+d_{i}\right) \\
& -2 m+i+1 \\
\geq & \left(d_{i}-1\right)\left(\frac{(a+\Delta) n-2}{a+b}-i(a+b+1)+2\right) \\
& -2 m+i+1 \\
\geq & \left(d_{i}-1\right)((i(a+b+1)+2 m-2)-i(a+b+1) \\
& +2)-2 m+i+1
\end{aligned}
$$




$$
=2 m\left(d_{i}-2\right)+i+1 \geq 3,
$$

a contradiction.

Therefore, the desired theorem is proved.

\section{Sharpness}

In this section, we aim to show that the independent set neighborhood union condition $\mid N_{G}\left(x_{1}\right) \cup N_{G}\left(x_{2}\right) \cup \cdots \cup$ $N_{G}\left(x_{i}\right) \mid \geq \frac{(b-\Delta) n+2}{a+b}+i$ in Theorem 3 is tight, i.e., we can't replace $\frac{(b-\Delta) n+2}{a+b}+i$ by $\frac{(b-\Delta) n+2}{a+b}+i-1$.

Let $m \geq 1, \Delta \geq 0, i \geq 2$ and $2 \leq a=b-\Delta$ be integers and $p$ is a positive integer which is assumed to be large enough. Let $G=K_{2 p a t} \vee(p b t) K_{2}$. Here two non-negative integer functions $g$ and $f$ are defined by $g(x)=a$ and $f(x)=b=$ $a+\Delta$ for each $x \in V(G)$. We ensure that $n=2 p t(a+b) \geq$ $\frac{(a+b)(i(a+b+1)+2 m-2)+2}{a+\Delta}, \delta(G)=2 p a t+1 \geq \frac{(i-1)(b-\Delta)(b+2)+1}{a+\Delta}+1$ and

$$
\begin{aligned}
& \frac{(b-\Delta) n+2}{a+b}+i-1 \\
< & \left|N_{G}\left(x_{1}\right) \cup N_{G}\left(x_{2}\right) \cup \cdots \cup N_{G}\left(x_{i}\right)\right| \\
= & 2 p a t+i=\frac{a n}{a+b}+i=\frac{(b-\Delta) n}{a+b}+i \\
< & \frac{(b-\Delta) n+2}{a+b}+i
\end{aligned}
$$

for any independent subset $\left\{x_{1}, x_{2}, \cdots, x_{i}\right\} \subseteq V(G)$. Set $S=V\left(K_{2 p a t}\right)$ and $T=V\left((p b t) K_{2}\right)$. We get $|S|=2 p a t$ and $|T|=a p b t$. Let $m=2 p b t$. For a $m$-matching $M=$ $(p b t) K_{2} \subset G$, let $H=G-M$. We obtain $d_{H-S}(T)=0$ and $\varepsilon(S, T)=2$. Hence, we infer

$$
\begin{aligned}
& f(S)+d_{H-S}(T)-g(T) \\
= & f(S)-g(T)=b(2 p a t)-a(2 p b t) \\
= & 0<2=\varepsilon(S, T) .
\end{aligned}
$$

From Theorem 4, we know that $H$ doesn't have a fractional $(g, f)$-factor containing a given edge $e \in E(H)$, i.e., $G$ doesn't have a fractional $(g, f)$-factor containing $e$, and exclude $M$. That is to say, the independent set neighborhood union condition $\left|N_{G}\left(x_{1}\right) \cup N_{G}\left(x_{2}\right) \cup \cdots \cup N_{G}\left(x_{i}\right)\right| \geq \frac{(b-\Delta) n+2}{a+b}+i$ in Theorem 3 is best in some sense.

\section{Conclusion}

The graph model is the most commonly used framework in computer networks. By representing the network as a graph, the corresponding network problem can be transformed into a graph problem, and the knowledge of graph theory can be used to analyze and solve the corresponding graph problems. This note focuses on the data transmission problem in the network, and uses the fractional factor theory to analyze the feasibility of data transmission in the network under certain conditions. The independent set neighborhood union condition reflects the basic organizational structure of the network and is an important parameter for network designing. We present an independent set neighborhood union condition for a graph admits including a fractional $(g, f)$-factor containing given edge $e$ and excluding a $m$-matching. This result indicates that under the condition where $m$ station doesn't intersect channels blocking or damaging, a certain range of data can still be transmitted between stations, and the route can pass through a specified channel. Furthermore, the presented conclusion has important guiding significance for the scientists in the network designing stage of data transmission networks. From a theoretical point of view, the independent set neighborhood union bound obtained in this note is tight.

Conflict of Interests The authors hereby declare that there is no conflict of interests regarding the publication of this paper.

Acknowledgement: We thank the reviewers for their insightful comments in the improvement of the paper. The work has been partially supported by a postdoctoral research grant in Jiangsu province (1701128B) and a Postdoctoral Research Grant of China (2017M621690).

\section{References}

[1] Bondy J.A., Murty U.S.R., Graph Theory, 2008, Springer, Berlin.

[2] Anstee R., An algorithmic proof of Tutte's $f$-factor theorem, J. Algorithms, 1985, 6, 112-131.

[3] Li Z., Yan G., Zhang X., On fractional ( $g, f)$-deleted graphs, Math. Appl. (in Chinese), 2003, 16, 148-154.

[4] Gao W., Gao Y., Toughness condition for a graph to be a fractional $(g, f, n)$-critical deleted graph, Sci. World J., 2014, 369798, http://dx.doi.org/10.1155/2014/369798.

[5] Gao W., Liang L., Xu T.W., Zhou J.X., Tight toughness condition for fractional $(g, f, n)$-critical graphs, J. Korean Math. Soc., 2014, 51, 55-65.

[6] Gao W., Wang W.F., New isolated toughness condition for fractional $(g, f, n)$-critical graphs, Colloq. Math., 2017, 147, 55-66.

[7] Gao W., Liang L., Chen Y.H., An isolated toughness condition for graphs to be fractional $(k, m)$-deleted graphs, Utilitas Math., 2017, 105, 303-316. 
[8] GaoW., Guirao J.L.G.,Wu H.L., Two tight independent set conditions for fractional $(g, f, m)$-deleted graphs systems, Qual. Theory Dyn. Syst., 2018, 17, 231-243.

[9] Gao W., Guirao J.L.G., Abdel-Aty M., Xi W.F., An independent set degree condition for fractional critical deleted graphs, Discrete Cont. Dyn. S.-S, 2019, 12, 877-886.

[10] Gao W., Dimitrov D., Abdo H., Tight independent set neighborhood union condition for fractional critical deleted graphs and ID deleted graphs, Discrete Cont. Dyn. S.-S, 2019, 12, 711-721.

[11] Gao W., Wang W.F., A tight neighborhood union condition on fractional $(g, f, n 0, m)$-critical deleted graphs, Colloq. Math., 2017, 147, 291-298.

[12] Gao W., Zhang Y.Q., Chen Y.J., Neighborhood condition for all fractional $(g, f, n 0, m)$-critical deleted graphs, Open Phys., 2018, 16, 544-553.

[13] Zhou S.Z., Remarks on orthogonal factorizations of digraphs, Int. J. Comput. Math., 2014, 91, 2109-2117.

[14] Zhou S.Z., Sun Z.R., Xu Z.R., A result on $r$-orthogonal factorizations in digraphs, Eur. J. Combin., 2017, 65, 15-23.

[15] Zhou S.Z., Sun Z.R., Neighborhood conditions for fractional $I D k$-factor-critical graphs, Acta Math. Appl. Sinica, Engl. Series, 2018, 34, 636-644.
[16] Zhou S.Z., Zhang T., Some existence theorems on all fractional $(g, f)$-factors with prescribed properties, Acta Mathematicae Applicatae Sinica, English Series, 2018, 34, 344-351.

[17] Zhou S.Z., Yang F., Xu L., Two sufficient conditions for the existence of path factors in graphs, Scientia Iranica, DOI: 10.24200/SCI.2018.5151.1122

[18] Zhou S.Z., Some results about component factors in graphs, RAIRO-Oper. Res., DOI: 10.1051/ro/2017045

[19] Khellat F., Khormizi M.B., A global solution for a reaction diffusion equation on bounded domains, Appl. Math. Nonl. Sc., 2018, 3, 1-14.

[20] Lokesha V., Shruti R., Deepika T., Reckoning of the dissimilar topological indices of human liver, Appl. Math. Nonl. Sci., 2018, 3, 265-276.

[21] Pandey P.K., Jaboob S.S.A., A finite difference method for a numerical solution of elliptic boundary value problems, Appl. Math. Nonl. Sc., 2018, 3, 311-320.

[22] Li Z., Yan G., Zhang X., On fractional $(g, f)$-covered graphs, OR Trans. (in Chinese), 2002, 6, 65-68. 